Lengua y Sociedad, revista de lingüística teórica y aplicada

Vol. 16, n. ${ }^{\circ}$ 2, Lima, julio-diciembre de 2017, pp. 125-140 https://doi.org/10.15381/lengsoc.v16i2.22378

\title{
La inserción del fonema /ř/ en el quechua actual: una explicación desde una teoría de las clases naturales $^{1}$
}

\section{The / $\check{l} /$ phoneme insertion in current Quechua: an explanation from a theory of natural classes}

\author{
Norma Meneses Tutaya \\ UNMMSM/CILA/DALIN \\ nmenesest@unmsm.edu.pe
}

\begin{abstract}
Resumen
En el presente estudio se plantea una explicación probable de la inserción de la vibrante múltiple prepalatal /ř/ como un segmento «natural» en el inventario fonológico del quechua actual pese a la evidencia de que se deriva del contacto con el español. Es decir, que el fonema /ř/ ha sido incorporado al sistema fonológico de algunos dialectos quechuas y está en proceso de estarlo en otros, debido fundamentalmente a que corresponde a una posición «naturalmente aceptable» dentro del sistema del quechua más que a un préstamo o a una interferencia del español andino. En todo caso, sin dejar de lado ambas explicaciones, planteo que el factor determinante es intrínseco al sistema fonológico del quechua y que esto se explica desde un enfoque que denomino teoría de las clases naturales. Por este concepto, el sistema consonántico del quechua está organizado en subsistemas de clases naturales donde prevalece la clase [+cons, +cor] en cuyo interior se produce la oposición entre las subclases [+cons, +cor, +ant] / [+cons, +cor, + post]. La presencia de la vibrante múltiple retrofleja se explicaría por su reubicación en la segunda subclase donde rellena una casilla vacía.
\end{abstract}

Palabras clave: fonología quechua, fonema vibrante múltiple, clases y subclases naturales, teoría de las clases naturales.

\begin{abstract}
The present study brings a possible explanation of how vibrant multiple prepalatal /ř/ is a «natural» segment in consonantal inventory of current Quechua despite its evident derivation from the contact with Spanish. In other words, the phoneme /ř / has been incorporated into the phonological system of some dialects of Quechua and it is in process of expansion to all dialects, due mainly to its correspondance to a «natural» slot within the Quechua system rather than a merely loan explanation or an interference of the Andean Spanish. In any case, without neglecting any of the given explanations, I propose that the determinant factor is intrinsic to the phonological system of Quechua from the point of view of a natural classes theory. Because of this theory, Quechua consonantal system is organized into natural classes, where [+cons, + cor $]$ class is nuclear. Within this natural class there is an opposition between two subclasses: [+cons, +cor, +ant] / [+cons, +cor, + post]. The presence of the multiple retroreflex vibrant would be explained by their relocation in the second subclass where it fullfills an empty cell.
\end{abstract}

Keywords: Quechua phonology, multiple vibrant phoneme, natural classes and subclasses, natural classes theory. 


\section{Introducción}

Los estudios de descripción gramatical de los diversos dialectos del quechua actual con excepción del quechua de Junín nos reportan la presencia del fonema vibrante simple /r/ en el inventario fonológico quechua como propio del mismo, a excepción del dialecto de Junín, que presenta únicamente la lateral alveolar. En contraste, la presencia del segmento vibrante múltiple /ř/ es reportado en contados dialectos aunque se observa su creciente uso en otros. Cuando ocurre la presencia de este segmento, es reconocido solo como parte de un préstamo lexical del castellano, tal como lo son las consonantes oclusivas sonoras y las vocales e y o.

Por otro lado, todos los investigadores coinciden también en la peculiar pronunciación de este segmento; sin embargo, no hay unanimidad en su descripción. Parker (1976) lo describe como un fricativo sonoro retroflejo, pues parece producirse con una imperceptible retroflexión del ápice de la lengua, Taylor (1996) lo describe igual, como fricativo retroflejo. Quesada (1976) es el único que lo discrimina como un fonema vibrante alveopalatal múltiple y no le reconoce el rasgo retroflejo. Coombs (1976) y los restantes elaboradores de las gramáticas editadas por el IEP y el Ministerio de Educación en 1976 reconocen este segmento como vibrante alveolar múltiple retroflejo. Convengo con el planteamiento de Quesada (1976) de que se trata de una vibrante múltiple que se realiza en un punto muy cercano al palatal y que no es de carácter retroflejo; por ello, prefiero denominarlo vibrante múltiple prepalatal. Propongo que la pronunciación peculiar se debe a una cuasi fricativización del segmento vibrante múltiple debido a que su cercanía al punto palatal hace que se fuerce una disminución de la vibración de este segmento hasta casi el nivel de una fricción. Esto explica la propuesta de Parker (1976) y Taylor (1996) de reconocerlo como segmento fricativo retroflejo. Cabe mencionar que algunos estudiosos caracterizan este segmento como vibrante múltiple «rehilante», lo cual resulta discutible por la propia definición de rehilamiento ${ }^{2}$, pues no tiene sentido proponer que un segmento vibrante adquiere rasgo vibrante, si lo posee.

Para demostrar nuestra hipótesis de que la presencia de esta vibrante múltiple prepalatal y su inesperada pronunciación obedece a causas «naturales» dentro del sistema fonológico del quechua, recurrimos al contraste de los inventarios

2 Gabriel G. Bes (1964) nos informa que Amado Alonso empleó este término en 1925 para referirse a la vibración que acompaña a la articulación de algunas fricativas sonoras como la /y/ en la zona rioplatense de Argentina.

\section{Lengua y Sociedad}


consonánticos de ocho dialectos: tres norteños (Lambayeque, Cajamarca y San Martín), tres centrales (Ancash, Huánuco y Junín) y dos sureños (Cusco y Ayacucho). Luego analizamos los segmentos en base al modelo de rasgos para establecer las llamadas clases naturales en el inventario. Es decir, se trató de caracterizar los inventarios consonánticos en términos de clases naturales identificables como subsistemas dentro del sistema fonológico de esta lengua.

El marco teórico empleado es la propuesta de una teoría de las clases naturales que propongo para este análisis. Esta teoría se fundamenta en el modelo de rasgos y en el concepto de las clases naturales de Chomsky y Halle (1968) en Sound Pattern of English (SPE). Planteo extender a nivel de teoría el concepto de las clases naturales porque permite aplicar un análisis sistémico que puede servir para proponer explicaciones sobre algunos fenómenos aún insolubles en procesos de contacto lingüístico y en procesos de reanálisis de segmentos prestados, tal como es el caso de la vibrante múltiple prepalatal en el quechua.

La teoría de las clases naturales es una propuesta de base estructural y funcional ya que se percibe el sistema fonológico de una lengua como una estructura diasistémica donde uno de su subsistemas tiene un rol nuclear. Las conclusiones iniciales de este estudio pueden servir como una propuesta para reincidir en el carácter sistémico de todos los componentes de la gramática de una lengua. Es decir, que la lengua y cada componente funcionan como un sistema de sistemas a manera de elementos fractales.

\section{La presencia de los segmentos $/ \mathrm{r} / \mathrm{y} / \mathrm{r} /$ en los dialectos quechuas}

La revisión de las descripciones fonológicas de los diversos quechuas se realizó tanto de textos específicos de estudios fonológicos, como de los capítulos dedicados a la descripción fonológica en las gramáticas de estos dialectos. A continuación transcribimos los inventarios consonánticos de los dialectos quechuas norteños, centrales y sureños. Cabe aclarar que con fines de análisis, en algunos casos, se ha cambiado ligeramente la representación de los segmentos empleando una forma homogénea para todos los quechuas, por ejemplo /tg/ por /č/. 


\section{Norma Meneses Tutaya}

\subsection{Los dialectos norteños}

Se presentan los inventarios consonánticos del quechua de Ferreñafe en Lambayeque, de Cajamarca y de San Martín.

Tabla 1. Inventario consonántico del quechua de Ferreñafe, Lambayeque

\begin{tabular}{l|l|l|l|l|l|l}
\hline \multicolumn{1}{r|}{ Punto } & Bilabial & Labiodental & Apical & Palatal & Velar & Uvular \\
\hline Oclusiva & $/ \mathrm{p} /$ & & $/ \mathrm{t} /$ & & $/ \mathrm{k} /$ & $/ \mathrm{q} /$ \\
\hline Sonorizada & $\mathrm{b}$ & & $\mathrm{d}$ & & $\mathrm{g}$ & \\
\hline Fricativa & & (f) & $/ \mathrm{s} /$ & $/ \check{\mathrm{s}} /$ & $(\mathrm{x})$ & \\
\hline Fricativa sonora & & & & $/ \check{\mathrm{z} /}$ & & \\
\hline Frica. son. retro. & & & & $/ \check{\mathrm{r}} /$ & & \\
\hline Africada & & & & $/ \check{c} /$ & & \\
\hline Africada retro. & & & & $/ \hat{\mathrm{c}} /$ & & \\
\hline Lateral & & & $/ \mathrm{l} /$ & & & \\
\hline Vibrante & & & $/ \mathrm{r} /$ & & & \\
\hline Semiconsonante & $/ \mathrm{w} /$ & & & $/ \mathrm{y} /$ & & \\
\hline Nasal & $/ \mathrm{m} /$ & & $/ \mathrm{n} /$ & $/ \mathrm{n} /$ & & \\
\hline
\end{tabular}

Fuente: G. Taylor, 1996.

Taylor (1996, p. 24) reporta que el fonema /r/ es una vibrante simple apical que presenta como alófono a la vibrante múltiple en inicio de palabra en Incahuasi y como una fricativa retrofleja en Ayamachay: rruray 'hacer'. En esta última a veces se presenta en final de palabra: yawarr 'sangre'. También mantienen el contraste entre la vibrante simple y la vibrante múltiple en los préstamos del castellano: fyerru 'hierro' / fyeru 'feo'.

Tabla 2. Inventario consonántico del quechua de Cajamarca

\begin{tabular}{l|l|l|l|l|l|l}
\hline \multicolumn{1}{r|}{ Punto } & Bilabial & Dental & Alveolar & Palatal & Velar & Uvular \\
\hline Oclusiva & $/ \mathrm{p} /$ & $/ \mathrm{t} /$ & & & $/ \mathrm{k} /$ & $/ \mathrm{q} /$ \\
\hline Sonorizada & $\mathrm{b}$ & $\mathrm{d}$ & & & $\mathrm{g}$ & \\
\hline Fricativa & $(\phi)$ & $/ \mathrm{s} /$ & & $/ \check{\mathrm{s}} /$ & $(\mathrm{x})$ & \\
\hline Fricativa retrofleja & & & & $/ \mathrm{s} /$ & & \\
\hline
\end{tabular}


La inserción del fonema /ř / en el quechua actual

\begin{tabular}{|c|c|c|c|c|c|c|}
\hline Modo $\quad$ Punto & Bilabial & Dental & Alveolar & Palatal & Velar & Uvular \\
\hline Africada & & & & $|\check{c}|$ & & \\
\hline Africada sonora & & & & $/ \mathrm{j} /$ & & \\
\hline Africada retrofleja & & & & $\mid \hat{c} /$ & & \\
\hline Lateral & & /1/ & & & & \\
\hline Vibrante & & $/ \mathrm{r} /$ & & & & \\
\hline Vibrante retrofleja & & & & $\mid \check{\mathrm{r}} /$ & & \\
\hline Semiconsonante & /w/ & & & $/ \mathrm{j} /$ & & \\
\hline Nasal & $/ \mathrm{m} /$ & $/ \mathrm{n} /$ & & $/ \mathrm{n} /$ & & \\
\hline
\end{tabular}

Fuente: F. Quesada, 1976.

Quesada (1976, p. 42) postula que la vibrante simple proviene del protoquechua porque se encuentra en todas las posiciones dentro de la palabra y aparece en todos los dialectos quechuas, excepto el de Junín. Además indica que la vibrante alveopalatal retrofleja se encuentra en un proceso de fonemización en este dialecto pues presenta pares mínimos: saru 'apareamiento' y sarru 'pisar'.

Tabla 3. Inventario consonántico del quechua de San Martin

\begin{tabular}{|c|c|c|c|c|c|c|c|}
\hline Modo Punto & Bilabial & $\begin{array}{l}\text { Labio- } \\
\text { dental }\end{array}$ & Dental & Alveolar & Palatal & Velar & Uvular \\
\hline Oclusiva & $/ \mathrm{p} /$ & & $/ \mathrm{t} /$ & & & /k/ & \\
\hline sonorizada & (b) & & (d) & & & (g) & \\
\hline Fricativa & & (f) & $/ \mathrm{s} /$ & & | & $(\mathrm{x})$ & \\
\hline Africada & & & & & $\mid \check{c} /$ & & \\
\hline Lateral & & & (1) & & $/ \Lambda /$ & & \\
\hline Vibrante & & & $/ \mathrm{r} /$ & & & & \\
\hline Vibrante múltiple & & & (r) & & & & \\
\hline Semiconsonante & $/ \mathrm{w} /$ & & & & $/ y /$ & & \\
\hline Nasal & $/ \mathrm{m} /$ & & $/ \mathrm{n} /$ & & $/ \mathrm{n} /$ & & \\
\hline
\end{tabular}

( ) préstamo del español. Fuente: D. Coombs, 1976.

Coombs (1976, p. 31) establece que la vibrante múltiple aparece solo en préstamos del español. 


\section{Norma Meneses Tutaya}

\subsection{Las variedades centrales}

A continuación se presentan los inventarios consonánticos de las variedades centrales de Huamalíes y Dos de Mayo, Huánuco, de Ancash y de Junín.

Tabla 4. Inventario consonántico del quechua de Huamalíes y Dos de Mayo, Huánuco

\begin{tabular}{|c|c|c|c|c|c|c|}
\hline Modo Punto & Bilabial & Alveolar & $\begin{array}{l}\text { Post } \\
\text { alveolar }\end{array}$ & Palatal & Velar & Uvular \\
\hline Oclusiva & $/ \mathrm{p} /$ & $/ \mathrm{t} /$ & & & $/ \mathrm{k} /$ & /G/ \\
\hline Fricativa & & /s/ & & /šs/ & $\mid \mathrm{x} /$ & \\
\hline Africada & & & & $\mid \check{c} /$ & & \\
\hline Lateral & & /1/ & & & & \\
\hline Vibrante & & $/ \mathrm{r} /$ & & & & \\
\hline Vibrante retrofleja & & & & $\mid \check{r} /$ & & \\
\hline Semiconsonante & & & & 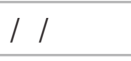 & /w/ & \\
\hline Nasal & $/ \mathrm{m} /$ & $/ \mathrm{n} /$ & & $/ \mathrm{n} /$ & & \\
\hline
\end{tabular}

Fuente: A. Tucto, 20.

Tabla 5. Inventario consonántico del quechua de Ancash

\begin{tabular}{|c|c|c|c|c|c|c|}
\hline Modo Punto & Bilabial & Alveolar & Palatal & Velar & Uvular & Glotal \\
\hline Oclusiva & $/ \mathrm{p} /$ & $/ \mathrm{t} /$ & & $/ \mathrm{k} /$ & /q/ & \\
\hline préstamo & (b) & (d) & & (g) & & \\
\hline Fricativa & $(\phi)$ & /s/ & $|\check{S}|$ & & & $/ \mathrm{h} /$ \\
\hline Africada sord. & & $/ \mathrm{c} /$ & $\mid \check{c} /$ & & & \\
\hline Africada son. & & & $/ \tilde{\mathrm{j}} /$ & & & \\
\hline Africada retro. & & & $/ \hat{\mathrm{c}} /$ & & & \\
\hline Lateral & & /1/ & $/ \Lambda /$ & & & \\
\hline Vibrante & & $/ \mathrm{r} /$ & & & & \\
\hline Vibrante & & & $(\check{\mathrm{r}})$ & & & \\
\hline Semiconsonante & $/ \mathrm{w} /$ & & $/ \mathrm{j} /$ & & & \\
\hline Nasal & $/ \mathrm{m} /$ & $/ \mathrm{n} /$ & /n/ & & & \\
\hline
\end{tabular}

Fuente: G. Parker, 1976. 
Parker (1976, p. 4) reporta que la vibrante simple /r/ tiene una realización como fricativa retrofleja sonora /ř / en posición inicial y final de palabra y como vibrante simple en posición intermedia.

Tabla 6. Inventario consonántico del quechua de Junín

\begin{tabular}{|c|c|c|c|c|c|c|}
\hline Modo Punto & Bilabial & Alveolar & Palatal & Velar & Uvular & Glotal \\
\hline Oclusiva & $/ \mathrm{p} /$ & $/ \mathrm{t} /$ & & $/ \mathrm{k} /$ & /q/ & \\
\hline Préstamo & (b) & (d) & & (g) & & \\
\hline Oclusiva africada & & & $\mid \check{c} /$ & & & \\
\hline Oclusiva afric. retro. & & & $/ \hat{\mathrm{c}} /$ & & & \\
\hline Fricativa & $(\phi)$ & /s/ & /šl & & & $/ \mathrm{h} /$ \\
\hline Lateral & & /1/ & $\mid \Lambda /$ & & & \\
\hline Vibrante & & $/ \mathrm{r} /$ & & & & \\
\hline Vibrante retrofleja & & $(\check{r})$ & & & & \\
\hline Semiconsonante & /w/ & & $/ \mathrm{j} /$ & & & \\
\hline Nasal & $/ \mathrm{m} /$ & $/ \mathrm{n} /$ & $/ \mathrm{n} /$ & & & \\
\hline
\end{tabular}

Fuente: R. Cerrón, 1976.

Cerrón (1976, pp. 48-49) define al fonema /r/vibrante simple como un segmento fonológico recientemente incorporado en su sistema como producto de un préstamo de palabras de alguna variedad de quechua sureño. La vibrante múltiple es considerada como un préstamo más reciente del castellano andino y solo está presente en las palabras de este origen. En buena medida se debe al proceso de bilingüismo que se realizó aceleradamente en esta región.

\subsection{Las variedades sureñas}

A continuación se presentan los inventarios consonánticos de las variedades del Cusco y Ayacucho. 


\section{Norma Meneses Tutaya}

Tabla 7. Inventario consonántico del quechua de Cusco

\begin{tabular}{|c|c|c|c|c|c|c|}
\hline Modo Punto & Bilabial & Alveolar & Palatal & Velar & Uvular & Glotal \\
\hline Oclusiva & $/ \mathrm{p} /$ & $/ \mathrm{t} /$ & & /k/ & /q/ & \\
\hline Oclusiva africada & & & $\mid \check{c} /$ & & & \\
\hline Oclusiva aspirada & $/ \mathrm{p}^{\mathrm{h}} /$ & $/ \mathrm{t}^{\mathrm{h}} /$ & $/ \check{c}^{h} /$ & $/ \mathrm{k}^{\mathrm{h}} /$ & $/ q^{h} /$ & \\
\hline Ocls. glotalizada & $/ \mathrm{p}^{\prime} /$ & $/ t^{\prime} /$ & $|\check{c} \prime|$ & $/ \mathrm{k}^{\prime} /$ & /q'/ & \\
\hline préstamo & (b) & (d) & & (g) & & \\
\hline Fricativa & $(\phi)$ & $/ \mathrm{s} /$ & & & & $/ \mathrm{h} /$ \\
\hline Lateral & & /1/ & $/ \Lambda /$ & & & \\
\hline Vibrante & & $/ \mathrm{r} /$ & & & & \\
\hline Vibrante retrofleja & & & $(\check{r})$ & & & \\
\hline Semiconsonante & & & $/ \mathrm{j} /$ & & & \\
\hline Nasal & $/ \mathrm{m} /$ & $/ \mathrm{n} /$ & $/ \mathrm{n} /$ & & & \\
\hline
\end{tabular}

Fuente: A. Cusihuamán, 1976.

Tabla 8. Inventario consonántico del quechua de Ayacucho

\begin{tabular}{|c|c|c|c|c|c|c|}
\hline Modo Punto & Labial & Alveolar & Palatal & Velar & Postvelar & Glotal \\
\hline Oclusiva & $/ \mathrm{p} /$ & $/ \mathrm{t} /$ & & $/ \mathrm{k} /$ & /q/ & \\
\hline Oclusiva africada & & & $|\check{c}|$ & & & \\
\hline préstamo & (b) & (d) & & (g) & & \\
\hline Fricativa & (f) & /s/ & & & & $/ \mathrm{h} /$ \\
\hline Lateral & & /1/ & $/ \Lambda /$ & & & \\
\hline Vibrante & & $/ \mathrm{r} /$ & & & & \\
\hline $\begin{array}{l}\text { Vibr. múltipl. } \\
\text { retro }\end{array}$ & & $(\check{\mathrm{r}})$ & & & & \\
\hline Semiconsonante & $/ \mathrm{w} /$ & & $/ \mathrm{j} /$ & & & \\
\hline Nasal & $/ \mathrm{m} /$ & $/ \mathrm{n} /$ & $/ \mathrm{n} /$ & & & \\
\hline
\end{tabular}

Fuente: C. Soto, 1976. 


\section{La teoría de las clases naturales: una concepción sistémica del inventario fonológico}

Empleo el concepto de clases naturales de Chomsky y Halle (1968), quienes lo establecen como parte de su teoría de los rasgos en SPE, para asignarle una aplicación distinta a la que originalmente fue destinada. Como se sabe, las clases naturales forman parte de la explicación de un proceso fonológico. En su modelo, una regla toma la forma como se observa al final de este párrafo. La generalización de la clase natural se construye por reglas restrictivas que solo se aplican a clases naturales de sonidos. Esto es, que el conjunto de sonidos que se rigen por la regla que se especifica a la izquierda de la flecha, que es descrito con los dos rasgos de -cont y -son, constituyen una clase natural y, por extensión, a cualquier conjunto de sonidos que presenten ambos rasgos formará parte de esta clase natural.

$$
\left[\begin{array}{c}
\text {-cont } \\
\text {-son }
\end{array}\right] \rightarrow[+ \text { voice }] /[+ \text { nasal }]
$$

En su texto, Bisol (2005) expone el concepto de clases naturales y hace hincapié en la necesidad de que los rasgos que distinguen a un conjunto de segmentos como pertenecientes a una clase natural deben ser relevantes como instrumento caracterizador de una clase natural. Indica que dos o más segmentos constituyen una clase natural cuando el número necesario de rasgos para definirlos como clase es menor al número de rasgos necesarios para definir a cada miembro de la misma. En otras palabras, que los rasgos caracterizadores de la clase funcionen como un «mínimo común divisor» de rasgos caracterizadores.

Bisol (2005) también nos alcanza la propuesta de Hyman (1975, pp. 139-140) con la cual plantea los criterios para identificar las clases naturales. Este autor establece que dos o más segmentos forman una clase natural cuando uno o más de los siguientes criterios se evidencia en alguna lengua:

- Los dos segmentos siguen las mismas reglas fonológicas.

- Los dos segmentos funcionan juntos en los mismos ambientes de las reglas fonológicas.

- Un segmento es convertido en el otro mediante una regla fonológica.

- Un segmento es derivado en el ambiente del otro segmento (como en los casos de asimilación). 
A continuación, se presentan las clases naturales presentes en los inventarios consonánticos del quechua. Postulo que existen cuatro clases naturales bastante bien definidas en el quechua las cuales son: la clase de +obstruyente, la clase + coronal y la clase + nasal. Considero que estas clases se distribuyen en tres de los cuatro planos articulatorios básicos, los cuales corresponden a los cuatro movimientos simultáneos de los músculos que intervienen en el proceso articulatorio: a) modo de articulación (movimiento de control de la salida del aire), b) punto de articulación (movimiento de ubicación de los articuladores en el aparato fonador, c) salida del aire (movimiento de la úvula para controlar el pasaje de salida del aire hacia los orificios nasales), y d) sonoridad (movimiento de las cuerdas vocales para determinar si un sonido se produce con o sin vibración en la glotis). El rasgo de la sonoridad no es de mucha utilidad en quechua pues este contraste no tiene carácter distintivo como ocurre en las lenguas europeas. Su empleo es más bien como un punto de articulación bastante laxo en algunas variedades. En el cusqueño se da como un proceso complementario de espirantización y glotalización de oclusivas.

a. Plano del modo de articulación (movimiento de control de la salida del aire):

1. La clase natural de los segmentos con el rasgo +obstruyente. Cabe notar que no forma un grupo de oposición o contraste con los segmentos que comparten el rasgo -obstruyente o +continuo en quechua como ocurre en muchas lenguas europeas.

Tabla 7. Clase natural de los segmentos del quechua con el rasgo +obstruyente

\begin{tabular}{l}
\hline \multicolumn{1}{c}{ +obstruyente } \\
\hline$/ \mathrm{p} /$ \\
\hline$/ \mathrm{t} /$ \\
\hline$/ \mathrm{c} /$ \\
\hline$/ \mathrm{k} /$ \\
\hline$/ \mathrm{q} /$
\end{tabular}

Fuente: Elaboración propia.

b. Plano del punto de articulación (movimiento de ubicación de los 
articuladores):

1. La clase natural de los segmentos con el rasgo + coronal. Forman el grupo mayoritario de segmentos en el inventario consonántico de los diversos dialectos quechuas.

1.1. Se subdivide en dos subclases naturales por los rasgos contrastados +anterior y + posterior que dan lugar a varios pares consonánticos opuestos como se evidencia en el siguiente cuadro, el cual sintetiza los segmentos hallados en los dialectos revisados. Por esta razón, se puede establecer que este contraste es el núcleo estructural del sistema fonológico del quechua. Se pudo separar las dos subclases y establecerlos como clases naturales, pero no lo hice así porque deseo hacer notar la relación intrínseca entre las dos subclases por la cual finalmente tienen una posición gravitante en el sistema fonológico del quechua.

\begin{tabular}{|c|c|}
\hline \multicolumn{2}{|c|}{ +coronal } \\
\hline +anterior & +posterior \\
\hline \multicolumn{2}{|l|}{$/ \mathrm{t} /$} \\
\hline \multirow[t]{2}{*}{$/ \mathrm{c} /$} & $\mid \check{c} /$ \\
\hline & $/ \hat{\mathrm{c}} /$ \\
\hline \multirow[t]{2}{*}{ /s/ } & /š/ \\
\hline & $/ \hat{\mathrm{s}} /$ \\
\hline /1/ & $/ \Lambda /$ \\
\hline \multicolumn{2}{|l|}{$/ \mathrm{r} /$} \\
\hline & $/ \mathrm{j} /$ \\
\hline$/ \mathrm{n} /$ & /n/ \\
\hline
\end{tabular}

Fuente: Elaboración propia.

1.2. La decisión de reconocer una sola clase natural +coronal y su posterior subdivisión, nos permitió visualizar la existencia de otra subclase interna a la subclase +coronal + posterior: la referida a la presencia o ausencia del 
rasgo distribuido. Cuando es -distribuido el rasgo es reconocido como retroflejo. En el siguiente cuadro se evidencia lo expuesto.

Tabla 9. Subclase natural de los segmentos del quechua con el rasgo +coronal +posterior

\begin{tabular}{l|l}
\hline \multicolumn{2}{c}{+ coronal +posterior } \\
\hline \multicolumn{1}{c}{+ +distribuido } & \multicolumn{1}{c}{-distribuido } \\
\hline$/ \check{c} /$ & $/ \hat{\mathrm{c}} /$ \\
\hline & \\
\hline$/ \check{\mathrm{s}} /$ & $/ \hat{\mathrm{s}} /$ \\
\hline & $/ \Lambda /$ \\
\hline & \\
\hline$/ \mathrm{j} /$ & \\
\hline$/ \mathrm{n} /$ & \\
\hline
\end{tabular}

Fuente: Elaboración propia.

2. La clase natural de los segmentos con rasgo -coronal. Comprende a aquellos que no se producen con la intervención de la parte flexible de la lengua y tienen una articulación en la zona periférica dentro de la cavidad bucal supraglótica. Esta clase se subdivide en dos grupos -coronal +anterior $\mathrm{y}$-coronal + posterior, donde el primer grupo corresponde a la articulación labial y el segundo a las articulaciones velar, uvular, tal como se presenta en cuadro siguiente. Cabe precisar que estos dos grupos no se encuentran en oposición por un único rasgo pues no se hallan pares consonánticos que así lo evidencien.

Tabla 10. Clase natural de los segmentos con rasgo -coronal

\begin{tabular}{l|l}
\hline \multicolumn{2}{c}{-coronal } \\
$\begin{array}{c}\text { +anterior } \\
\text { +labial }\end{array}$ & \multicolumn{1}{c}{$\begin{array}{c}\text { +posterior } \\
+ \text { dorsal }\end{array}$} \\
\hline$/ \mathrm{p} /$ & \multicolumn{1}{|c}{} \\
\hline$/ \mathrm{w} /$ & $/ \mathrm{q} /$ \\
\hline & \\
\hline$/ \mathrm{m} /$ & \\
\hline
\end{tabular}

Fuente: Elaboración propia. 
c. Plano de la salida del aire (movimiento de la úvula)

1. La clase natural de los segmentos nasales. Se forma por el empleo de la cavidad nasal como salida o escape del aire empleado para la dicción. Se encuentra en contraste con la gran mayoría de segmentos que tienen una salida por el orificio bucal. En quechua no forma un grupo de oposición directa con un grupo preciso de salida oral.

Tabla 11. Clase natural de los segmentos nasales

\begin{tabular}{ll}
\hline \multicolumn{1}{c}{+ nasal } \\
\hline$/ \mathrm{m} /$ \\
\hline$/ \mathrm{n} /$ \\
$\mathrm{n} /$
\end{tabular}

Fuente: Elaboración propia.

\section{La inserción del fonema /řr en el sistema fonológico del quechua como un segmento "natural»}

La aplicación del concepto clase natural al análisis de los inventarios consonánticos de estas variedades quechuas nos permite visualizar y explicar la razón por la cual el segmento / $\check{\mathrm{r}} / \mathrm{o} / \hat{\mathrm{r}} /$ ha sido «natural» y fácilmente incorporado en el sistema fonológico de casi todas las variedades quechua dentro del contexto del contacto con el español.

En el sistema consonántico del quechua existe como eje nuclear de su estructuración la diferencia de la subclase natural + coronal +anterior versus la subclase natural +coronal + posterior. Consideramos que la vibrante alveolar múltiple del español fue incorporada al sistema del quechua como un segmento prestado del español y reinterpretado como una vibrante prepalatal múltiple (quechua de Ayacucho) y en la mayoría de los casos como una vibrante prepalatal o palatal retrofleja debido a que en el eje +coronal +posterior existía hasta dos casilleros vacíos donde se podía ubicar cómoda y «naturalmente» el segmento vibrante alveolar múltiple del español. El primero fue como una vibrante prepalatal múltiple 
y el segundo como una vibrante retrofleja ya que en algunas variedades norteñas y centrales se observa la presencia de una articulación complementaria de retroflexión o -distribuido. El siguiente cuadro se muestra ambas posibilidades.

Tabla 12. Inserción del segmento /r/ en la subclase +coronal +posterior del quechua

\begin{tabular}{l|l}
\hline $\begin{array}{c}\text { +coronal } \\
\text { +anterior }\end{array}$ & \multicolumn{1}{|c}{$\begin{array}{c}\text { +coronal } \\
\text { +posterior }\end{array}$} \\
\hline$/ \mathrm{t} /$ & $/ \check{\mathrm{c}} /$ \\
\hline$/ \mathrm{c} /$ & $/ \hat{\mathrm{c}} /$ \\
\hline$/ \mathrm{s} /$ & $/ \check{\mathrm{s}} /$ \\
\hline & $/ \hat{\mathrm{s}} /$ \\
\hline$/ \mathrm{l} /$ & $/ \mathrm{L} /$ \\
\hline$/ \mathrm{r} /$ & $1 / \check{\mathbf{r}} /$ \\
\hline & $2 / \hat{\mathrm{r}} /$ \\
\hline & $/ \mathrm{j} /$ \\
\hline$/ \mathrm{n} /$ & $/ \mathrm{n} /$ \\
\hline
\end{tabular}

Fuente: Elaboración propia.

Aún queda por discernir el aspecto diacrónico de esta explicación pues con el cuadro anterior se evidencia la relativa facilidad con la cual el segmento prestado del español se ha ubicado «naturalmente» en la posición vacía; mas no la antigüedad de esta inserción. Se podría alegar que los primeros bilingües quechua-castellano durante la primera centuria del contacto andino-español, fueron quienes hicieron esta reinterpretación de la vibrante múltiple del español y generaron una vibrante prepalatal múltiple en su habla castellana lo cual dio origen al peculiar sonido retroflejo o «rehilante» que tiene este segmento en el castellano andino. Probablemente, este uso de la vibrante prepalatal múltiple ingresó, tiempo después, al quechua a través de los bilingües castellano andino-quechua y este fuera reinterpretado por los monolingües de las variedades norteñas y centrales como una vibrante retrofleja dado que en el sistema consonántico de algunas de ellas existe el rasgo complementario de retroflexión en los segmentos + coronal +posterior. En las variedades sureñas quedó como un segmento prepalatal o palatal vibrante múltiple para las 
palabras prestadas del español řusa 'rosa', řamu 'ramo'; incluso, ha formado así pares mínimos mixtos como quech. karu 'lejos' y esp. kařu 'carro'.

\section{Algunas conclusiones}

Se ha demostrado la utilidad del concepto de clases naturales para explicar el carácter sistémico de los inventarios consonánticos pues se puede observar que dentro de los mismos es posible hallar microsistemas o subsistemas consonánticos. Por extensión se puede argüir que se trata de una teoría de las clases naturales pues sus presupuestos teóricos se pueden aplicar con propiedad a otros ámbitos.

Mediante la aplicación de este análisis de clases naturales se ha podido jerarquizar los microsistemas consonánticos del quechua y establecer que el eje de la estructuración del sistema consonántico de esta lengua está constituida por la clase natural de los segmentos con rasgo + coronal, específicamente por la oposición interna entre + coronal +anterior versus + coronal + posterior.

Se hace evidente que el segmento vibrante prepalatal múltiple del castellano andino ha pasado prestado al quechua mediante una inserción fonológica del mismo como vibrante prepalatal múltiple o como vibrante prepalatal retrofleja, en razón de que existía un vacío en el sistema original para ambos sonidos.

Es posible que el análisis de clases naturales aplicado a los sistemas fonológicos en contacto como el caso del quechua y el castellano pueda explicar algunos enigmas como es el caso del surgimiento de la vibrante múltiple retrofleja en el castellano andino.

Las conclusiones expuestas son iniciales y se necesita más estudios para determinar no solo si las clases naturales hacen posible no solo la inserción de un segmento prestado de otra lengua como en el caso de la vibrante prepalatal del castellano andino /ř/ en el quechua actual; sino, además, si este se ha incorporado dentro del sistema con sus usos originales en la lengua española.

Otro tema que ha quedado para ser discutido más adelante es la aplicación de esta teoría a los estudios de lingüística histórica. En especial, al caso del estatus de la vibrante simple /r/ en el sistema fonológico del protoquechua. 


\section{Referencias Bibliográficas}

Alonso-Cortés, Á. (2002). Lingüística. Madrid: Cátedra.

Bisol, L. (Org.). (2005). Introdução a estudos de fonología do português brasileiro (4. ${ }^{\text {a }}$ ed.). Porto Alegre: EDIPUCRs.

Campbell, L. (1999). Historical Linguistics: An Introduction. Massachusetts: Massachusetts Institute of Technology.

Cerrón-Palomino, R. (1976). Gramática quechua Junín-Huanca. Lima: Instituto de Estudios Peruanos - Ministerio de Educación del Perú.

Cerrón-Palomino, R. (2003). Lingüística quechua. Cusco: C. E. R. A. «Bartolomé de las Casas».

Coombs, D. et al. (1976). Gramática quechua San Martín. Lima: Instituto de Estudios Peruanos - Ministerio de Educación del Perú.

Cusihuamán, A. (2001). Gramática quechua Cuzco-Collao. Cusco: C. E. R. A. «Bartolomé de las Casas».

Flemming, E. (2005). Deriving natural clases in phonology. Lingua, 115(3), 287-309. doi:10.1016/j.lingua.2003.10.005

Morales, A. (1992). Teorías delos rasgos distintivos. ASJU, 26(2), 613-643. Recuperado de https://www.ehu.eus/ojs/index.php/asju/article/viewFile/8309/7471

Parker, G. (1965). Gramática del quechua ayacuchano. Lima: Universidad Nacional Mayor de San Marcos.

Parker, G. (1976). Gramática quechua Áncash-Huailas. Lima: Instituto de Estudios Peruanos - Ministerio de Educación del Perú.

Quesada, F. (1976). Gramática quechua Cajamarca-Cañaris. Lima: Instituto de Estudios Peruanos - Ministerio de Educación del Perú.

Quesada, F. (2006). Quechua de Cajamarca. Morfología. Fonología. Sintaxis. Lima: Mantaro.

Soto, C. (1976). Gramática quechua Ayacucho-Chanca. Lima: Instituto de Estudios Peruanos - Ministerio de Educación del Perú.

Taylor, G. (1996). El Quechua de Ferreñafe. Cajamarca: Acku Quinde.

140 Lengua y Sociedad 